\title{
Proceedings of the winter meeting of the British Neuropsychiatric Association, London, 21 January 1994
}

The topic of the meeting was Brain repair and rehabilitation. Professor A Compston (Cambridge) opened the meeting with a talk on preparing to repair the brain. Growth factor conditions, the paucity of precursor cells for neurons and glia and the inhibition of axonal regeneration in the adult mammalian brain limit the extent of endogenous brain repair. In addition, brain repair strategies make little sense if the underlying degenerative process is not halted. Recent work suggests that growth factors also have a trophic and survival effect on neurons and glia in the developed brain and may therefore provide a suitable environment for brain repair. This has led to trials of the free radical scavenger $\alpha$-tocopherol (vitamin E) and the selective monoamine oxidase $\beta$-antagonist deprenyl in Parkinson's disease with encouraging results. When large numbers of cells have been damaged through the disease process, however, cell implantation may be required for neurotransmitter release and to restore connectivity.

Dr S Dunnet (Cambridge) dealt with strategies of brain repair through neural transplantation. He discussed the mechanisms by which neural grafts may exert functional effects. These include nonspecific consequences of surgery, acute and chronic release of deficient neurochemicals, bridging tissues for host regeneration, diffuse reinnervation of the host brain, and reciprocal graft-host reconnection. These mechanisms are not mutually exclusive and all have been seen to apply in different model systems. He illustrated his lecture with references to the neural implants carried out in Parkinson's disease.

Dr S Butler (Bristol) discussed neuroplasticity in the context of the unexpected consequences of spontaneous lesions in the central and peripheral nervous system in man or those experimentally induced in animals. Examples of such plasticity can be observed following lesions of sensory organs, such as the retina, which result in changes in cortical representation to include new areas outside those damaged, or following cortical lesions in, for example, the motor cortex, whose function is taken over by adjacent areas. In humans, similar observations can be made using magnetic stimulation of the cortex in amputees. Faulty reorganisation of cortical mapping may be central to the development of phantom limb.

Professor R Frackowiak (London) discussed functional reorganisation as a mechanism for recovered function following brain injury with reference to PET studies in stroke. Studies by his group have demonstrated that focal lesions in the internal capsule result in regional cerebral blood flow (rCBF) reduction in distant areas related to motor function such as the dorsolateral prefrontal cortex, inferior frontal cortex and contralateral cerebellum. An increase in rCBF occurs in the contralateral hemisphere in areas such as the posterior cingulate cortex subserving attention. In patients who recover hand function, finger movement is associated with bilateral increases in rCBF in cortical areas related to motor function to a much greater degree than that produced by finger movement of the unaffected hand. These studies provide firm evidence of large scale reorganisation of cortical function during recovery.

Dr P Eames (Buckinghamshire) dealt with episodic disorders after brain injury drawing on his experience of posttraumatic epilepsy and temporolimbic syndromes following head trauma. In his experience, both conditions are frequent, but temporolimbic syndromes may be overlooked more often. Under this rubric, he included the episodic dyscontrol syndrome, transient dysphoria, and brief psychotic and confusional states.

The last part of the meeting, devoted to the role of different disciplines in the process of neurorehabilitation, started with a contribution from $\mathrm{Dr} K$ Barrett (Keele University) who described his experience in setting up a neuropsychiatric brain injury rehabilitation service in North Staffordshire. His unit serves a population of 500000 and behavioural problems following head trauma in young adults are the main reason for admission. $\mathrm{Dr} G$ Beaumont (London) followed with an account of the contribution of clinical neuropsychologists to the rehabilitation of brain injured patients. The assessment of goals for rehabilitation and the choice of appropriate interventions were, in his view, the key contributions. Both required detailed knowledge of cognitive and other psychological processes, awareness or resources, and organisational skills. Finally, Dr R Greenwood (London) dealt with the neurological aspects of rehabilitation of brain injured patients. Based on his experience of rehabilitating severely brain injured patients, he favoured a problem oriented approach incorporating behavioural programmes and careful assessment of outcome. The meeting ended with a lively panel discussion, lead by Professor A Lishman, on who should do what in brain rehabilitation? M A RON
The National Hospital for Neurology and Neurosurgery, Queen Square,
Qu 